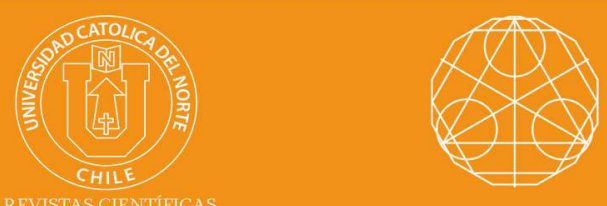

\title{
Construction of sequences of borderenergetic graphs
}

S. K. Vaidya* (iD orcid.org/0000-0002-5394-1424

K. M. Popat* (iD) orcid.org/0000-0002-3818-3717

*Saurashtra University, Rajkot, GJ, India.

samirkvaidya@yahoo.co.in

${ }^{* *}$ Atmiya University, Rajkot, GJ, India, India.

kalpeshmpopat@gmail.com

\section{Abstract:}

The graphs whose energy is same as that of complete graphs are known as borderenergetic graphs. We propose a procedure for the construction of borderenergetic graphs and investigate three sequences of borderenergetic graphs.

Keywords: Eigenvalues; Energy of Graphs; Borderenergetic Graphs.

\section{Cite this article as (IEEE citation style):}

S. Vaidya and K. Popat, "Construction of sequences of borderenergetic graphs", Proyecciones (Antofagasta, On line), vol. 38 , no. 4 , pp. $837-847$, Oct. 2019 , doi: $10.22199 /$ issn.07176279-2019-04-0055. [Accessed dd-mm-yyyy].

Article copyright: (c) 2019 Samir K. Vaidya and Kalpesh M. Popat. This is an open access article distributed under the terms of the Creative Commons Licence, which permits unrestricted use and distribution provided the original author and source are credited. 


\section{Introduction}

For standard terminology and notations in graph theory we follow West [1] while the terms related to algebra are used in sense of Lang [2]. We denote the complement of graph $G$ by $\bar{G}$, the complete graph on $p$ vertices by $K_{p}$, the null graph by $\overline{K_{p}}$. The join of $G_{1}$ and $G_{2}$ is a graph $G=G_{1} \vee G_{2}$ with vertex set $V\left(G_{1}\right) \cup V\left(G_{2}\right)$ and an edge set consisting of all the edges of $G_{1}$ and $G_{2}$ together with the edges joining each vertex of $G_{1}$ with every vertex of $G_{2}$. The line graph $L(G)$ of a graph $\mathrm{G}$ is the graph whose vertex set is $E(G)$ and two vertices are adjacent in $L(G)$ whenever they are incident in $G$.

Let $G$ be a connected undirected simple graph with vertex set $V(G)=$ $\left\{v_{1}, v_{2}, \cdots, v_{n}\right\}$. The adjacency matrix denoted by $A(G)$ of $G$ is defined to be $A(G)=\left[a_{i j}\right]$, such that, $a_{i j}=1$ if $v_{i}$ is adjacent with $v_{j}$, and 0 otherwise.

Let $\lambda_{1}, \lambda_{2}, \cdots \lambda_{n}$ be eigenvalues of $A(G)$. These eigenvalues form the spectrum $G$, which is denoted by $\operatorname{spec}(G)$. The energy of the graph $G$, denoted by $E(G)$, is defined as

$$
E(G)=\sum_{i=1}^{n}\left|\lambda_{i}\right|
$$

The concept of energy of graph was introduced by Gutman [5] in 1978. A brief account on energy of graph can be found in Cvetkovic [3] and $\mathrm{Li}$ [4].

The energy of complete graph $K_{n}$ is defined to be $2(n-1)$. The graphs of order $n$, whose energy exceeds than the energy of the complete graph $K_{n}$ are called hyperenergetic graphs otherwise the graphs of order $n$ with $E(G) \leq E\left(K_{n}\right)$, are called non-hyperenergetic. A curious question is: Are there graphs whose energy is equal to that of $K_{n}$ ? The search to answer this question provides the reason for initiation of a new concept.

Recently, Gong et al. [6] proposed the concept of borderenergetic graphs, according to him graphs of order $n$ for which $E(G)=2(n-1)$ are called borderenergetic graphs. Obviously, the complete graphs $K_{n}$ are borderenergetic. Hence, we are interested only in non complete borderenergetic graphs. Gong et al. [6] have proved that there exist no borderenergetic graph of order less than 7 . The following results were obtained by computer aided search

- There exist a unique borderenergetic graphs of order 7 [6]. 
- There are exactly 6 non-isomorphic borderenergetic graphs of order $8[6]$.

- There are exactly 17 non isomorphic borderenergetic graphs of order $9[6]$.

- There are exactly 49 borderenergetic graphs of order 10 [7].

- There are exactly 158 borderenergetic graphs of order 11 [8], of which 157 are connected.

- There are exactly 572 connected borderenergetic graphs of order 12 $[9]$.

We pose the following problem :

How to obtain a larger borderenergetic graph from a given borderenergetic graph?

To answer this question, let us understand the sequence of complete graph $\Omega=\left\{K_{1}, K_{2}, \cdots, K_{n}, \cdots\right\}$. The graph $K_{n}$ can be viewed as $K_{n}=$ $K_{n-1} \vee K_{1}$ as shown in following figure

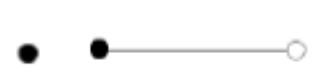

$K_{1} \quad K_{2}=K_{1} \vee K_{1}$

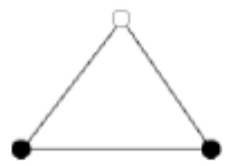

$K_{3}=K_{2} \vee K_{1}$

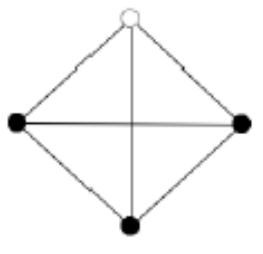

$K_{4}=K_{3} \vee K_{1}$

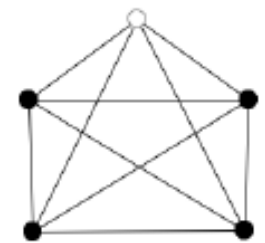

$K_{5}=K_{4} \vee K_{1}$

Figure 1: Sequence of Complete Graphs $K_{n}$

As the Borderenergetic graphs have the same energies as the complete graphs, then natural quest is : Can we employ the same logic for the construction of new borderenergetic graph from the given one? We got an affirmative answer of this question and obtain some infinite sequences of borderenergetic graphs.

Proposition 1.1. [3] If $G_{1}$ is $r_{1}$ regular with $n_{1}$ vertices and $G_{2}$ is $r_{2}$ regular with $n_{2}$ vertices then the characteristic polynomial of the join $G_{1} \vee$ $G_{2}$ is given by

$$
\phi\left(G_{1} \vee G_{2}, x\right)=\frac{\phi\left(G_{1}, x\right) \phi\left(G_{2}, x\right)}{\left(x-r_{1}\right)\left(x-r_{2}\right)}\left(\left(x-r_{1}\right)\left(x-r_{2}\right)-n_{1} n_{2}\right)
$$




\section{Main Results}

Theorem 2.1. Let $G$ be $r$ - regular borderenergetic graph with $n$ vertices then for $p \neq 0, G \vee \overline{K_{P}}$ is borderenergetic if and only if $p=n-r$.

Proof: Let $r=\lambda_{1}, \lambda_{2}, \cdots \lambda_{n}$ be the eigenvalues of $G$. As $G$ is borderenergetic of $n$ - vertices, $E(G)=2(n-1)$ which imply that

$$
\sum_{i=2}^{n}\left|\lambda_{i}\right|=2 n-2-r
$$

By Proposition 1.1, the characteristic polynomial of $G \vee \overline{K_{p}}$ is given by

$$
\phi\left(G \vee \overline{K_{p}}\right)=x^{p-1}\left(x-\lambda_{2}\right)\left(x-\lambda_{3}\right) \cdots\left(x-\lambda_{i}\right)\left(x^{2}-r x-n p\right)
$$

Let $\alpha_{1}$ and $\alpha_{2}$ are roots of polynomial $x^{2}-r x-n p$. It is easy to observe that $\alpha_{1}$ and $\alpha_{2}$ are of opposite sign. With out loss of generality we assume that

$$
\alpha_{1}>0, \alpha_{2}<0
$$

Also,

$$
\begin{gathered}
\alpha_{1}+\alpha_{2}=r \\
\alpha_{1} \cdot \alpha_{2}=-n p
\end{gathered}
$$

Here,

$$
\operatorname{spec}\left(G \vee \bar{K}_{p}\right)=\left(\begin{array}{ccccccc}
0 & \lambda_{2} & \lambda_{3} & \cdots & \lambda_{n} & \alpha_{1} & \alpha_{2} \\
p-1 & 1 & 1 & \cdots & 1 & 1 & 1
\end{array}\right)
$$

Hence,

$$
\begin{aligned}
E\left(G \vee \overline{K_{p}}\right) & =\sum_{i=2}^{n}\left|\lambda_{i}\right|+\left|\alpha_{1}\right|+\left|\alpha_{2}\right| \\
& =\sum_{i=2}^{n}\left|\lambda_{i}\right|+\alpha_{1}-\alpha_{2}
\end{aligned}
$$

If $G \vee \overline{K_{p}}$ is borderenergetic then

$$
E\left(G \vee \overline{K_{p}}\right)=2(n+p-1)
$$




$$
\begin{gathered}
\Leftrightarrow \alpha_{1}-\alpha_{2}=2 n+2 p-1-\sum_{i=2}^{n}\left|\lambda_{i}\right| \\
\Leftrightarrow \alpha_{1}-\alpha_{2}=2 n+2 p-2-2 n+2+r=2 p+r
\end{gathered}
$$

By $(2.2)$ and $(2.4)$

$$
\begin{aligned}
& \alpha_{1}=p+r \\
& \alpha_{2}=-p
\end{aligned}
$$

By (2.3)

$$
\begin{array}{ll}
\alpha_{1} \cdot \alpha_{2} & =-n p \\
\Leftrightarrow p(p+r) & =n p \\
\Leftrightarrow p^{2}-p(n-r) & =0 \\
\Leftrightarrow p & =n-r
\end{array}
$$

\section{Sequence of Borderenergetic Graphs}

In this section we construct an infinite sequence of borderenergetic graphs. To construct the sequence we take any $r$ - regular borderenergetic graphs of order $n$ as our base graphs and then the sequence is obtained by joining $n-r$ vertices at each iteration.

Let $G^{(0)}$ is any $r$ - regular borderenergetic graphs of order $n$. Consider an infinite sequence of graphs $\mathcal{G}=\left\{G^{(0)}, G^{(1)}, \cdots, G^{(k)}, \cdots\right\}$ such that each $G^{(k)}$, of order $n+k(n-r)$, satisfies

$$
\begin{array}{lr}
G^{(1)} & =G^{(0)} \vee \overline{K_{n-r}} \\
G^{(2)} & =G^{(1)} \vee \overline{K_{n-r}} \\
\vdots & \vdots \\
G^{(k)} & =G^{(k-1)} \vee \overline{K_{n-r}}
\end{array}
$$

Using the fact that, If $G_{1}$ is $r_{1}-$ regular with $n_{1}$ vertices and $G_{2}$ is $r_{2}-$ regular with $n_{2}$ vertices such that $r_{1}+n_{2}=r_{2}+n_{1}$ then $G_{1} \vee G_{2}$ is regular of degree $r_{1}+n_{2}=r_{2}+n_{1}$. We assert that, $G^{(k)}$ is $r+k(n-r)$ regular graph for any $k \geq 1$.

Lemma 3.1. Let $G^{(0)}$ be $r$ regular graph with eigenvalues $r=\lambda_{1}, \lambda_{2}, \cdots, \lambda_{n}$ then for any $G^{(k)} \in \mathcal{G}, k \geq 1$, the spectrum of $G^{(k)}$ is 


$$
\operatorname{spec}\left(G^{(k)}\right)=\left(\begin{array}{ccccccc}
0 & \lambda_{2} & \lambda_{3} & \cdots & \lambda_{n} & r+k(n-r) & -n+r \\
k(n-r-1) & 1 & 1 & \cdots & 1 & 1 & k
\end{array}\right)
$$

Proof: We prove this result by taking induction on $k$. From Theorem 2.1, it is clear that result is true for $k=1$.

Assume by mathematical induction that the result is true for $k=s-1$. Then by induction hypothesis

$$
\operatorname{spec}\left(G^{(s-1)}\right)=\left(\begin{array}{ccccccc}
0 & \lambda_{2} & \lambda_{3} & \cdots & \lambda_{n} & r+(s-1)(n-r) & -n+r \\
(s-1)(n-r-1) & 1 & 1 & \cdots & 1 & 1 & s-1
\end{array}\right)
$$

For $k=s, G^{(s)}=G^{(s-1)} \vee \overline{K_{n-r}}$. From Proposition 1.1,

$$
\begin{aligned}
\phi\left(G^{(s)}, x\right) & =x^{(s-1)(n-r-1)+(n-r-1)}\left(x-\lambda_{2}\right)\left(x-\lambda_{3}\right) \cdots\left(x-\lambda_{n}\right)(x+n-r)^{s-1} \\
& {\left[\left(x^{2}-(r+(s-1)(n-r) x-(n+(s-1)(n-r))(n-r)]\right.\right.} \\
& =x^{s(n-r-1)}\left(x-\lambda_{2}\right)\left(x-\lambda_{3}\right) \cdots\left(x-\lambda_{n}\right)(x-r-s(n-r))(x+n-r)^{s}
\end{aligned}
$$

Therefore,

$$
\operatorname{spec}\left(G^{(s)}\right)=\left(\begin{array}{ccccccc}
0 & \lambda_{2} & \lambda_{3} & \cdots & \lambda_{n} & r+s(n-r) & -n+r \\
s(n-r-1) & 1 & 1 & \cdots & 1 & 1 & s
\end{array}\right)
$$

Thus the result is true in this case as well hence by induction the result follows.

Theorem 3.2. For each $r \geq 1, G^{(k)}$ is non co-spectral and borderenergetic graphs with $K_{n+k(n-r)}$.

Proof: Clearly $G^{(k)}$, and $K_{n+k(n-r)}$ are non co-spectral.

$$
\begin{aligned}
E\left(G^{(k)}\right) & =\sum_{i=2}^{n}\left|\lambda_{i}\right|+r+k(n-r)+k(n-r) \\
& =2 n-2-r+r+2 k(n-r) \\
& =2(n+k(n-r)-1)=E\left(K_{n+k(n-r)}\right)
\end{aligned}
$$

\section{Sequences of Some Known Regular Borderenergetic Graphs}

In this section we construct three infinite class of regular borderenergetic graphs $\mathcal{H}_{i}=\left\{H_{i}^{(0)}, H_{i}^{(1)}, \cdots, H_{i}^{(k)}, \cdots\right\} \subseteq \mathcal{G}$ for $i=1,2,3$ by taking some known regular borderenergetic graphs as base graph. 


\subsection{The class $\mathcal{H}_{1}$}

Consider $H_{1}^{(0)}=$ Line graph of complete bipartite graph $K_{3,3}$ which 4 regular graph with 9 vertices

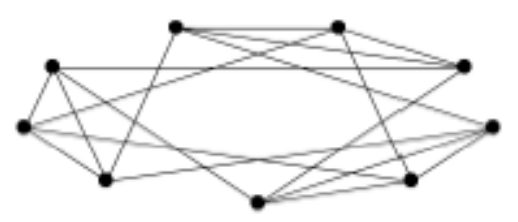

Figure 2: Line graph $K_{3,3}$

$$
\operatorname{spec}\left(H_{1}^{(0)}\right)=\left(\begin{array}{ccc}
4 & -2 & 1 \\
1 & 4 & 4
\end{array}\right), E\left(H_{1}^{(0)}\right)=16
$$

and thus it is borderenergetic with $K_{9}$. Consider an infinite sequence or borderenergetic graphs

$$
\mathcal{H}_{1}=\left\{H_{1}^{(0)}, H_{1}^{(1)}, H_{1}^{(2)}, \cdots, H_{1}^{(k)}, \cdots\right\}
$$

such that

$$
H_{1}^{(1)}=H_{1}^{(0)} \vee \overline{K_{5}}, H_{1}^{(2)}=H_{1}^{(1)} \vee \overline{K_{5}}, H_{1}^{(3)}=H_{1}^{(2)} \vee \overline{K_{5}} \cdots
$$

The parameters $n, r, E$ of the sequence of borderenergetic graphs are depicted in follwoing Table 1

\begin{tabular}{|c|c|c|c|c|c|}
\hline$G$ & $n$ & $r$ & spectra & $\boldsymbol{E}(\boldsymbol{G})$ & Borderenergetic With \\
\hline$H_{1}^{(0)}$ & 9 & 4 & $4^{1},(-2)^{4}, 1^{4}$ & 16 & $K_{9}$ \\
$H_{1}^{(1)}=H_{1}^{(0)} \vee K_{5}$ & 14 & 9 & $0^{4},(-2)^{4}, 1^{4}, 9^{1},(-5)^{1}$ & 26 & $K_{14}$ \\
\hline$H_{1}^{(2)}=H_{1}^{(1)} \vee \bar{K}$ & 19 & 14 & $0^{8},(-2)^{4}, 1^{4}, 14^{1},(-5)^{2}$ & 36 & $K_{19}$ \\
\hline$H_{1}^{(3)}=H_{1}^{(2)} \vee \overline{K_{5}}$ & 24 & 19 & $0^{12},(-2)^{4}, 1^{4}, 1^{1},(-5)^{3}$ & 46 & $K_{24}$ \\
\hline$H_{1}^{(4)}=H_{1}^{(3)} \vee \overline{K_{5}}$ & 29 & 24 & $0^{16},(-2)^{4}, 1^{4}, 24^{1},(-5)^{4}$ & 56 & $K_{29}$ \\
\hline$H_{1}^{(5)}=H_{1}^{(4)} \vee \overline{K_{5}}$ & 34 & 29 & $0^{20},(-2)^{4}, 1^{4}, 29^{1},(-5)^{5}$ & 66 & $K_{34}$ \\
\hline$\vdots$ & $\vdots$ & $\vdots$ & $\vdots$ & $\vdots$ & $\vdots$ \\
\hline
\end{tabular}




\subsection{The class $\mathcal{H}_{2}$}

Consider $H_{2}^{(0)}=$ Line graph of Petersen graph which is 4 regular graph with 15 vertices

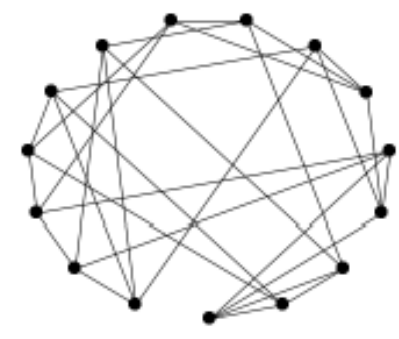

Figure 3: Line graph of Petersen Graph

As discussed in Gong et al. [6], the spectrum of the line graph of the Petersen graph is

$$
\operatorname{spec}\left(H_{2}^{(0)}\right)=\left(\begin{array}{cccc}
4 & -1 & 2 & -2 \\
1 & 4 & 5 & 5
\end{array}\right) E\left(H_{2}^{(0)}\right)=28
$$

and thus it is borderenergetic. Consider an infinite sequence or borderenergetic graphs

$$
\mathcal{H}_{2}=\left\{H_{2}^{(0)}, H_{2}^{(1)}, H_{2}^{(2)}, \cdots, H_{2}^{(k)}, \cdots\right\}
$$

such that

$$
H_{2}^{(1)}=H_{2}^{(0)} \vee \overline{K_{11}}, H_{2}^{(2)}=H_{2}^{(1)} \vee \overline{K_{11}}, H_{2}^{(3)}=H_{2}^{(2)} \vee \overline{K_{11}} \cdots
$$

The parameters $n, r, E$ of this sequence of borderenergetic graphs are depicted in following Table 1

Table 1

\begin{tabular}{|c|c|c|c|c|c|}
\hline$G$ & $n$ & $r$ & spectra & $E(G)$ & Borderenergetic With \\
\hline$H_{2}^{(0)}$ & 15 & 4 & $4^{1},(-1)^{4}, 2^{5},(-2)^{5}$ & 28 & $K_{15}$ \\
\hdashline$H_{2}^{(1)}=H_{2}^{(0)} \vee \bar{K}$ & 26 & 15 & $0^{10},(-1)^{4}, 2^{5},(-2)^{5}, 15^{1},(-11)^{1}$ & 50 & $K_{26}$ \\
\hline$H_{2}^{(2)}=H_{2}^{(1)} \vee \overline{K_{11}}$ & 37 & 26 & $0^{20},(-1)^{4}, 2^{5},(-2)^{5}, 26^{1},(-11)^{2}$ & 72 & $K_{37}$ \\
\hdashline$H_{2}^{(3)}=H_{2}^{(2)} \vee \overline{K_{11}}$ & 48 & 37 & $0^{30},(-1)^{4}, 2^{5},(-2)^{5}, 37^{1},(-11)^{3}$ & 94 & $K_{48}$ \\
\hdashline$H_{2}^{(4)}=H_{2}^{(3)} \vee \overline{K_{11}}$ & 59 & 48 & $0^{40},(-1)^{4}, 2^{5},(-2)^{5}, 48^{1},(-11)^{4}$ & 116 & $K_{59}$ \\
\hdashline$H_{2}^{(5)}=H_{2}^{(4)} \vee \overline{K_{11}}$ & 70 & 59 & $0^{50},(-1)^{4}, 2^{5},(-2)^{5}, 59^{1},(-11)^{5}$ & 138 & $K_{70}$ \\
\hline$\vdots$ & $\vdots$ & $\vdots$ & $\vdots$ & $\vdots$ & $\vdots$ \\
\hline$\vdots$ & $\vdots$ & $\vdots$ & $\vdots$ \\
\hline
\end{tabular}


Definition 4.1. The extended shadow graph $D_{2}^{*}(G)$ of a connected graph $G$ is constructed by taking two copies of $G$ say $G^{\prime}$ and $G^{\prime \prime}$. Join each vertex $u^{\prime}$ in $G^{\prime}$ to the neighbours of the corresponding vertex $u^{\prime \prime}$ and with $u^{\prime \prime}$ in $G^{\prime \prime}$.

Proposition 4.2. [10] Let $G$ be a graph with eigenvalues $\lambda_{1}, \lambda_{2}, \cdots, \lambda_{n}$ then the eigenvalues of $D_{2}^{*}(G)$ are $2 \lambda_{i}+1,(1 \leq i \leq n),(-1)$ (2n times).

\subsection{The class $\mathcal{H}_{3}$}

Consider $H_{3}^{(0)}=D_{2}^{*}\left(K_{n, n}\right)$ which is $2 n+1$ regular graph with $2 n$ vertices.

$$
\operatorname{spec}\left(H_{3}^{(0)}\right)=\left(\begin{array}{cccc}
2 n+1 & -2 n+1 & 1 & -1 \\
1 & 1 & 2 n-2 & 2 n
\end{array}\right) E\left(H_{3}^{(0)}\right)=8 n-2
$$

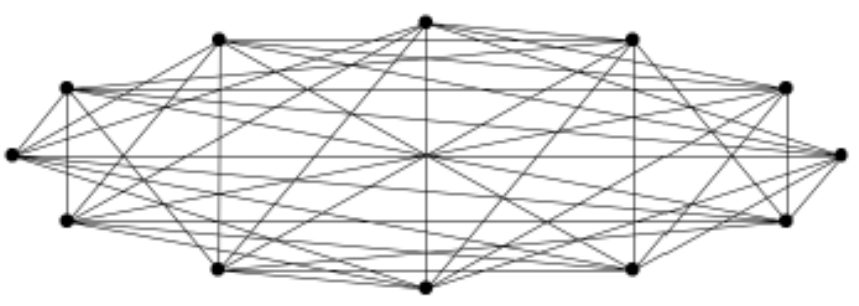

Figure 4: $D_{2}^{*}\left(K_{3,3}\right)$

and thus it is borderenergetic with $K_{4 n}$. Consider an infinite sequence or borderenergetic graphs

$$
\mathcal{H}_{3}=\left\{H_{3}^{(0)}, H_{3}^{(1)}, H_{3}^{(2)}, \cdots, H_{3}^{(k)}, \cdots\right\}
$$

such that

$$
H_{3}^{(1)}=H_{3}^{(0)} \vee \overline{K_{2 n-1}}, H_{3}^{(2)}=H^{(1)} \vee \overline{K_{2 n-1}}, H_{3}^{(3)}=H_{3}^{(2)} \vee \overline{K_{2 n-1}} \cdots
$$

The parameters $n, r, E$ of this sequence of borderenergetic graphs are depicted in follwoing Table 3 
Table 2

\begin{tabular}{|c|c|c|c|c|c|}
\hline$G$ & $n$ & $r$ & spectra & $E(G)$ & Borderenergetic With \\
\hline$H_{3}^{(0)}$ & $4 n$ & $2 n+1$ & $(2 n+1)^{1},(-2 n+1)^{1}, 1^{2 n-2},(-1)^{2 n}$ & $8 n-2$ & $K_{4 n}$ \\
\hline$H_{3}^{(1)}=H_{3}^{(0)} \vee \overline{K_{\mathrm{s}}}$ & $6 n-1$ & $4 n$ & $0^{2 n-2},(-2 n+1)^{2}, 1^{2 n-2},(-1)^{2 n},(4 n)^{1}$ & $12 n-4$ & $K_{\mathrm{en}-1}$ \\
\hline$H_{3}^{(2)}=H_{3}^{(1)} \vee \overline{K_{5}}$ & $8 n-2$ & $6 n-1$ & $0^{4 n-4},(-2 n+1)^{3}, 1^{2 n-2},(-1)^{2 n},(6 n-1)^{1}$ & $16 n-6$ & $K_{8 n-2}$ \\
\hline$H_{s}^{(3)}=H_{3}^{(2)} \vee \overline{K_{5}}$ & $10 n-3$ & $8 n-2$ & $0^{6 n-8},(-2 n+1)^{4}, 1^{2 n-2},(-1)^{2 n},(8 n-2)^{1}$ & $20 n-8$ & $K_{10 m-3}$ \\
\hline$H_{3}^{(4)}=H_{3}^{(9)} \vee K_{5}$ & $12 n-4$ & $10 n-3$ & $0^{\beta n-8},(-2 n+1)^{5}, 1^{2 n-2},(-1)^{2 n},(10 n-3)^{1}$ & $24 n-10$ & $K_{12 n-4}$ \\
\hline$H_{s}^{(8)}=H_{3}^{(4)} \vee \overline{K_{5}}$ & $14 n-5$ & $12 n-4$ & $0^{10 n-10},(-2 n+1)^{6}, 1^{2 n-2},(-1)^{2 n},(12 n-4)^{1}$ & $28 n-12$ & $K_{14 m-5}$ \\
\hline $\begin{array}{ll}n \\
\vdots\end{array}$ & ! & - & $\begin{array}{c}\vdots \\
\vdots\end{array}$ & +- & $\bar{\vdots}$ \\
\hline
\end{tabular}

\section{Concluding Remarks}

The survey of the existing literature on graph energy reveals that only handful graphs are proved to be borderenergetic graphs while we have constructed an infinite sequence of such graphs. This work will certainly add a new dimension in the field of graph energy.

\section{Acknowledgement}

The authors thank the anonymous referees for their valuable suggestions leading to the improvement of the original manuscript.

\section{References}

[1] D. West, Introduction to graph theory, 2nd ed. Chennai, TN: Pearson India, 2000.

[2] S. Lang, Algebra, 3rd ed., vol. 211. New York, NY: Springer, 2002, doi: 10.1007/978-1-4613-0041-0.

[3] D. Cvetkovic, P. Rowlinson and Simic Slobodan, An introduction to the theory of graph spectra, vol. 75. Cambridge: Cambridge University Press, 2010, doi: 10.1017/CB09780511801518.

[4] X. Li, Y. Shi, and I. Gutman, Graph energy. New York, NY: Springer, 2012, doi: 10.1007/978-1-4614-4220-2. 
[5] I. Gutman, "The energy of a graph", Berichte der mathematischstatistischen sektion im forschungszentrum graz, vol. 103, pp. $1-22,1978$.

[6] S. Gong, X. Li, G. Xu, I. Gutman and B. Furtula, "Borderenergetic graphs", MATCH Communications in mathematical and in computer chemistry, vol. 74, no. 2, pp. 321-332, 2015. [On line]. Available: https://bit.ly/2J5EfvW

[7] X. Li, M. Wei and S. Gong, "A computer search for the borderenergetic graphs of order 10", MATCH Communications in mathematical and in computer chemistry, vol. 74, no. 2, pp.333-342, 2015. [On line]. Available: https://bit.ly/35SQntZ

[8] Z. Shao, F. Deng, Correcting the number of borderenergetic graphs of order 10, MATCH Communications in mathematical and in computer chemistry, vol. 75, no. 2pp. 263-265, 2016. [On line]. Available: https://bit.ly/2qqbio4.

[9] B. Furtula and I. Gutman, "Borderenergetic graphs of order 12", Iranian journal of mathematical chemistry, vol. 8, no. 4, pp. 339343, 2017, doi: 10.22052/IJMC.2017.87093.1290.

[10] S. Vaidya, K. Popat, "Some borderenergetic and equienergetic graphs of arbitrarily large order", Communicated. 